Published in final edited form as:

Curr Treat Options Neurol. 2015 August ; 17(8): 362. doi:10.1007/s11940-015-0362-5.

\title{
Treatment of Primary Progressive Aphasia
}

\author{
Donna C. Tippett ${ }^{1,2,3}$, Argye E. Hillis ${ }^{1,3,4}$, and Kyrana Tsapkini ${ }^{1,{ }^{*}}$ \\ ${ }^{1}$ Department of Neurology Johns Hopkins University School of Medicine, Baltimore MD, USA \\ ${ }^{2}$ Department of Otolaryngology-Head and Neck Surgery, Johns Hopkins University School of \\ Medicine, Baltimore MD, USA \\ ${ }^{3}$ Department of Physical Medicine \& Rehabilitation, Johns Hopkins University School of Medicine, \\ Baltimore MD, USA \\ ${ }^{4}$ Department of Cognitive Science, Johns Hopkins University, Baltimore MD, USA
}

\begin{abstract}
Primary progressive aphasia (PPA) is a neurodegenerative disease that primarily affects language functions and often begins in the fifth or sixth decade of life. The devastating effects on work and home life call for the investigation of treatment alternatives. In this paper, we present a review of the literature on treatment approaches for this neurodegenerative disease. We also present new data from two intervention studies we have conducted, a behavioral one and a neuromodulatory one using transcranial direct current stimulation (tDCS) combined with written production intervention. We show that speech-language intervention improves language outcomes in individuals with PPA; and especially in the short term, tDCS augments generalization and maintenance of positive language outcomes. We also outline current issues and challenges in intervention approaches in PPA.
\end{abstract}

\section{Introduction}

Primary progressive aphasia (PPA) is a neurodegenerative syndrome that mainly affects language abilities including word finding, word usage, word comprehension, and sentence construction ${ }^{1-3}$. PPA is characterized by insidious onset and gradual deterioration of language associated with atrophy of the frontal and temporal regions of the left hemisphere ${ }^{1,4}$. In this neurodegenerative condition, language is disproportionately impaired for at least two years, without impairment in other cognitive domains other than praxis ${ }^{5}$. PPA is comprised of three main variants, each with specific clinical features and pathophysiology: nonfluent agrammatic PPA, semantic variant PPA, and logogpenic variant PPA ${ }^{3,6}$. Difficulty naming is an early and persistent impairment common to all three variants of PPA ${ }^{7-9}$.

\footnotetext{
"Corresponding author: Kyrana Tsapkini, PhD, Department of Neurology, Johns Hopkins Medical Institutions, 600 N. Wolfe Street, Phipps 488, Baltimore, MD 21237, USA, Tel: (+) 1-410-614-2646. tsapkini@jhmi.edu (Kyrana Tsapkini).

Compliance with Ethics Guidelines

Human and Animal Rights and Informed Consent This article does not contain any studies with human or animal subjects performed by any of the authors.
} 
Nonfluent agrammatic PPA (nfaPPA) is characterized by core features of agrammatic language production and/or apraxia of speech ${ }^{10-12}$. Spoken modality-specific naming impairments are reported in nfaPPA ${ }^{13}$ as are naming deficits specific to impaired naming of actions rather than objects ${ }^{13-15}$. Individuals with nfaPPA may become mute early in their disease progression ${ }^{16}$ and develop clinical features of parkinsonism and related syndromes, such as corticobasal syndrome or progressive supranuclear palsy ${ }^{17}$. Imaging abnormalities are present in left posterior frontal and insular regions $10,18,19$. The pathology is typically a tau-opathy, such as corticobasal degeneration, progressive supranuclear palsy, or frontotemporal lobar degeneration-tau ${ }^{3}$.

Semantic variant (svPPA) is defined by marked anomia and single-word comprehension deficits across input and output modalities ${ }^{20}$. Individuals with svPPA may display progressively impaired object naming, with preserved naming of actions, and greater difficulty in the written versus spoken modality, although both modalities are compromised ${ }^{14,15}$. This variant is associated with atrophy in ventrolateral anterior temporal lobes bilaterally, usually greater atrophy on the left 10,19 . Speech fluency, syntax, and word repetition are relatively preserved ${ }^{10}$. Individuals with svPPA also manifest behavioral symptoms as their disease progresses 21,22 . The pathology is most often frontotemporal lobar degeneration-TDP- $43^{3}$.

Logopenic variant (lvPPA) is distinguished by word retrieval and phrase and sentence repetition deficits. Single word comprehension and speech articulation are relatively spared ${ }^{3,23}$. Generalized cognitive decline, including language abilities, attention, memory, and visuospatial skills, is manifested over time ${ }^{24}$. Imaging abnormalities are seen in the left temporoparietal junction ${ }^{10,19}$. The pathology is usually Alzheimer's disease ${ }^{3}$.

Due to its onset in middle age, PPA profoundly impacts work and home life. Behavioral interventions - mainly for spoken naming - have been described to remediate the language deficits in PPA ${ }^{25-29}$. Word production impairments (both in oral and written modalities as manifested in deficits in picture naming and spelling) have important clinical value in PPA since they are the two earliest symptoms, thus allowing for early detection and intervention. Word finding and fluency difficulties are among the first symptoms in logopenic (lvPPA) and non-fluent (nfaPPA) variants ${ }^{30}$. Spelling is also impaired early in every subtype, and may predict the PPA subtype early in the course of the disease ${ }^{31}$. For example, surface dysgraphia symptoms are usually found in semantic variant (svPPA) or lvPPA, but more rarely in nfaPPA. Those with nfaPPA sometimes rely on spelling when they eventually become mute ${ }^{3}$.

\section{Cognitive Mechanisms Underlying Spoken and Written Word Production and Implications for Therapy}

In this section, we review the cognitive mechanisms involved in spoken and written production since spelling, naming and reading deficits are among the first and most disruptive symptoms in PPA, and their remediation is the goal of most interventions. Figure 1 shows the close relationship between spoken and written word production mechanisms in models of cognitive architecture. Specifically, word representations in either the written or spoken modality may be accessed from the other modality or the semantic (word meaning) 
system ${ }^{32,33}$. The implication, which is the basis of several treatment studies in post-stroke

aphasia ${ }^{34,35}$, is that both lexical and sublexical routes from one modality may contribute to word retrieval in the other. Thus, behavioral treatments stimulating residual knowledge across the semantic, phonological, and orthographic domains have resulted in cross-domain improvements $36-39$. For example, a combination of spelling treatment with spoken repetition $37,40,41$ improved written and spoken production even in participants with semantic impairments.

\section{Spoken and written production intervention studies in PPA}

Intervention studies in PPA are, in general, difficult due to the degenerative nature of the disease, the variable rate of decline among individuals and the inherent heterogeneity of each variant. For example, individuals with nfaPPA decline more rapidly in action than object naming, while those with svPPA show the opposite pattern; and those with svPPA show most notable decline in object semantics ${ }^{44}$. Therefore, most intervention studies are case reports or include a small number of participants (for a review see Croot et al., $2009^{45}$ ). Behavioral studies -across all PPA subtypes- have mostly investigated treatment of word retrieval: (a) semantic variant PPA (svPPA) ${ }^{46-50}$; b) non-fluent/agrammatic variant PPA (nfaPPA) ${ }^{51-54}$; and, c) logopenic variant PPA (lvPPA) ${ }^{28,55,56}$. These studies have shown encouraging results of language therapy, i.e., potential for new lexical learning in svPPA ${ }^{57}$ and lvPPA ${ }^{28}$, benefit of implementing errorless strategies ${ }^{50}$, the importance of early intervention ${ }^{57}$, and, potential for generalizability and retention of therapy gains ${ }^{28,56}$. Long-term effects of therapy gains are either not systematically examined or outcomes were variable when examined. For example, Meyer and colleagues ${ }^{58}$ reported response to repetition and reading/writing therapy for anomia in four individuals with PPA. They found significant improvements in some, but not, all treatment conditions over five months.

Intervention studies in individuals with apraxia of speech (AOS) associated with nfaPPA, characterized by syntactic disorders and apraxia of speech (AOS) in the initial stages, have targeted the single-word level. A recent study used reading of multisyllabic words as an intervention strategy in PPA with lasting and generalizable results. ${ }^{61}$

Only two behavioral studies of which we are aware have examined treatment of written language in PPA - one treating sublexical mechanisms ${ }^{30}$ and the other treating lexical processes ${ }^{28}$. Both treatments were successful, but long-term follow-up was examined only in one study 28 and was successful only for treated items. These studies have shown that results with language therapy alone are encouraging although limited, either because they have not shown generalization to untrained items or because they have lacked follow-up to evaluate the sustainability of therapy gains.

\section{Transcranial Direct Current Stimulation to Augmenting Language Interventions}

Transcranial Direct Current Stimulation (tDCS) has been identified as a promising intervention to augment behavioral treatment benefits in language therapy programs, mostly in stroke ${ }^{59-61}$ and Alzheimer's disease (AD). The benefits of tDCS- its low expense, high safety profile, and non-invasive nature-justify research on its use in PPA as a possible means to augment behavioral intervention effects and reduce the rate of decline in language. 
The precise mechanisms of tDCS are unknown; however, it is thought that tDCS changes the membrane potentials of neurons in a relatively focal area of brain tissue under the skull ${ }^{64-66}$. Anodal stimulation increases the likelihood of neural firing ${ }^{65}$. tDCS induces a subthreshold polarization of neurons too weak to generate action potentials, but sufficient to modulate the neuronal response threshold. Thus, tDCS alters the spontaneous firing rate of neurons to modulate their response to afferent signals ${ }^{67}$. These changes in response threshold correlate with task performance. Thus, increases or decreases in cortical excitability induced by tDCS are believed to promote long-term potentiation (LTP) and long-term depression (LTD). The changes in brain networks may include recruitment of undamaged areas of the brain to assume functions of damaged areas during language tasks ${ }^{68}$.

The positive effects of tDCS in motor and higher cognitive functions-including languagehave been identified in studies of healthy controls. After a single tDCS session, participants experienced improved performance lasting up to five hours; however, long-lasting effects of tDCS have been documented only in studies with repeated consecutive tDCS sessions ${ }^{60,69-71}$. Besides these proof-of-concept studies of healthy controls' motor skill learning ${ }^{72,73}$, there is a recent proof-of-concept study of verbal word learning ${ }^{74}$ confirming memory formation and consolidation after repeated consecutive sessions. The clinical importance of tDCS requires establishing therapy generalization and maintenance of treatment in clinical populations. Two research groups have provided relevant evidence: Fridriksson's group in spoken naming remediation in post-stroke aphasia $70,75,76$ and Boggio's group in associative memory remediation in $\mathrm{AD}^{77-79}$. After five consecutive stimulations, therapy gains were found to last up to four weeks $62,75,77,80$. The brain mechanisms that induce such effects are thought to be late long-term potentiation ${ }^{72}$ and/or protein synthesis ${ }^{73}$ that may constitute the physiological basis of long-term memory formation and offline consolidation ${ }^{73}$. Given that long-lasting learning reflects synaptic connectivity changes, the effects of tDCS are expected to be manifested in connectivity changes between nodes of neural networks. Indeed, studies that have looked at effects of tDCS on functional connectivity using resting-state fMRI (rsfMRI) have found significant changes in healthy controls ${ }^{81-83}$.

\section{tDCS Interventions in Neurodegenerative Disease}

tDCS has been shown to enhance cortical excitability and function ${ }^{84-86}$ when anodal current is applied in healthy individuals. Tasks employed in these studies include fluency, interference, picture naming, verbal learning, and proper noun learning. In clinical populations, tDCS has been used mainly to improve motor and language recovery, primarily after stroke ${ }^{59-61,86-93}$ A wide range of tasks have been targeted in post-stroke aphasia including: verb naming ${ }^{89}$, auditory verbal working memory ${ }^{93}$, repetition of syllables and words for treatment of speech apraxia ${ }^{94}$, word retrieval or picture naming for anomia treatment $61,70,75,88,92,95$. Despite the plethora of reports on language recovery using tDCS after stroke, only a few studies have examined it in neurodegenerative diseases (see recent reviews ${ }^{96,97}$ ): three studies on $\mathrm{AD}^{62,77,98}$, including only one study in which tDCS was applied for more than one session (five sessions) ${ }^{80}$ and which showed greater improvement with tDCS vs. sham in a visual recognition task (9\% vs. $2.6 \%)$ but without any 
task performed during either tDCS or sham conditions, two studies on frontotemporal dementia (FTD) ${ }^{99,100}$ (one session only with no effect of tDCS ${ }^{99}$ but also no task practiced during treatment, and 10 sessions with more improvement over tDCS vs. sham ${ }^{100}$ coupled with an oral naming task), and ours in PPA ${ }^{101}$ where (after 15 treatment sessions coupled with a spelling task) we found greater improvement with tDCS vs. sham (35\% of patients made significant improvement on untrained words with tDCS vs. $16 \%$ of patients made significant improvement on untrained words with sham). The tasks used during tDCS were verbal and visual recognition memory in $\mathrm{AD}$, spoken verbal fluency and naming in FTD, and spelling in our study in PPA. One FTD study ${ }^{99}$ did not find any effect of tDCS in improving verbal fluency which may have been because there was only one 40-minute stimulation session that was not coupled with language therapy. Other studies that did not couple tDCS with language therapy have repeatedly yielded no improvement in both healthy and patient populations $102-105$. A highly consistent finding across studies using a wide array of tasks is that tDCS-induced facilitation is highly dependent on the task subjects perform during stimulation and that tDCS-only conditions are consistently unsuccessful ${ }^{102-104,106 .}$

We are aware of only three other neuromodulation studies in PPA; all three used repetitive transcranial magnetic stimulation (rTMS) ${ }^{107-109}$, and all showed improvement with neuromodulation during language therapy tasks. Of particular interest is Trebbastoni's et al. case study 108 in which after TMS stimulation during five consecutive sessions twice (interleaved with five sham stimulations) over the dorsolateral prefrontal cortex and close to the inferior frontal gyrus (IFG) and middle frontal gyrus (MFG), the PPA participant showed improvement in phonemic verbal fluency and written language (decrease of semantic and syntactic errors in sentences).

Long-term benefits of neuromodulation (tDCS or TMS) have not been clearly identified; this is especially true for neurodegenerative disease ${ }^{64}$. One study in AD showed improvement in naming one month after tDCS ${ }^{78}$. In general, long-term effects-whenever shown-appeared after at least five consecutive days of stimulation. Determining the duration of therapeutic effects is critical, especially in neural degeneration, because it enables more effective planning of whether and when treatment should be repeated. In both recent studies using tDCS in PPA 100,101, long-term effects (up to two months) have been identified, offering promise of the proposed intervention as a tool of slowing down the rate of decline in neurodegeneration.

\section{Generalization of Treatment Gains to other Language and Cognitive Functions}

In addition to generalization to untrained items, generalization to untrained tasks is expected, and sometimes observed when trained and untrained tasks share cognitive functions 34,35 . Two studies in post-stroke aphasia evaluated tDCS effects of training oral naming ${ }^{95}$ and syllable-word repetition ${ }^{87}$ and have shown generalization to written naming. In published interventions in PPA, these effects are not fully investigated. Future studies should test the hypothesis that gains from training both spoken and written word representations will generalize to related language and cognitive functions. Furthermore, since language and cognitive impairments associated with PPA interfere with activities in 
daily life and life satisfaction, future studies should evaluate how improvements in language and cognitive functions enhance quality of life for individuals with PPA and their families.

\section{Medications}

Because the most common pathology underlying lvPPA is Alzheimer's disease pathology, the decline in symptoms might be reduced with cholinesterase inhibitors and/or memantine, medications that have been shown to somewhat reduce the rate of decline in cognition in clinically diagnosed Alzheimer's disease. However, a large randomized clinical trial specifically in lvPPA has not been completed. Case studies have reported improvement in language with steroid treatment ${ }^{110}$ or Omentum Transposition Therapy ${ }^{111}$, but these effects have not been replicated. Theoretically, medications that enhance neuroplasticity, such as selective serotonin reuptake inhibitors, might augment the effects of tDCS, but the combination of interventions has not been studied in PPA.

\section{Conclusions: Challenges and New Venues}

The present review shows that language interventions are possible and can be successful in a neurodegenerative disease. All behavioral interventions in PPA cited above showed improvement of the language function targeted. However, not all of them showed generalizable and long-lasting effects. Many reasons may be responsible for these findings: heterogeneity of symptoms and pathologies reflected by the different PPA variants, different stages of disease progression at baseline, variable rate of decline between participants and studies. Neuromodulation with tDCS offers promise as a means of augmenting language therapy to improve written language function at least temporarily in PPA. The consistent finding of generalization of treatment benefits to untreated items and the superior sustainability of treatment effects with tDCS justifies further investigations. To date there are only a few studies 101,112 with small sample sizes, so results require caution in interpretation but offer hope for improved outcomes of combined language therapy and tDCS. Future interventions need to address particular challenges, such as ways to account for the variable effect of degeneration in each individual, generalization of treatment to other cognitive functions, impact and improvement in quality of life of the individuals with PPA. Longitudinal studies also need to determine whether interventions have the potential of altering the rate of disease progression or even slowing down the progression of symptoms for some time. Future research is needed to determine whether medications, used alone or in combination with speech and language treatment with or without neuromodulation, can be of benefit in reducing the rate of language decline in PPA. Finally, future research should address the brain mechanisms involved in both behavioral and neuromodulatory interventions.

\section{Acknowledgments}

Some of the research reported in this paper, as well as the authors of the paper, were supported by NIDCD through R01 14129060, R01 DC011317, R01 DC03681, R01 DC014475 and by the Science of Learning Institute of Johns Hopkins University.

\section{References}

Papers of particular interest, published recently, have been highlighted as: 
- Of importance (papers critical to the classification and differential diagnosis of the disease)

•• Of major importance (papers relevant to interventions in PPA)

1*. Mesulam MM. Primary progressive aphasia. Ann Neurol. 2001; 49(4):425-432. Seminal paper delineating the characteristics of PPA. [PubMed: 11310619]

2*. Mesulam M, Weintraub S. Primary progressive aphasia and kindred disorders. Handb Clin Neurol. 2008; 89:573-587. Follow-up and update paper with differential diagnosis of PPA. [PubMed: 18631780]

3*. Gorno-Tempini ML, Hillis AE, Weintraub S, et al. Classification of primary progressive aphasia and its variants. Neurology. 2011; 76(11):1006-1014. Most recently used classification criteria for the diagnosis of PPA: state-of-the-art. [PubMed: 21325651]

4. Mesulam MM, Wieneke C, Hurley R, et al. Words and objects at the tip of the left temporal lobe in primary progressive aphasia. Brain. 2013; 136(Pt 2):601-618. [PubMed: 23361063]

5*. Mesulam MM. Slowly progressive aphasia without generalized dementia. Ann Neurol. 1982; 11(6):592-598. First paper describing characteristics of PPA even before this name was given. [PubMed: 7114808]

6. Josephs KA, Duffy JR. Apraxia of speech and nonfluent aphasia: A new clinical marker for corticobasal degeneration and progressive supranuclear palsy. Curr Opin Neurol. 2008; 21(6):688692. [PubMed: 18989114]

7*. Grossman M, Ash S. Primary progressive aphasia: A review. Neurocase. 2004; 10(1):3-18. Critical review of the state-of-the art of PPA at the time of publication. [PubMed: 15849155]

8. Hurley RS, Paller KA, Wieneke CA, et al. Electrophysiology of object naming in primary progressive aphasia. J Neurosci. 2009; 29(50):15762-15769. [PubMed: 20016092]

9. Mesulam MM. Primary progressive aphasia and the language network: The $2013 \mathrm{H}$. houston merritt lecture. Neurology. 2013; 81(5):456-462. [PubMed: 23897873]

10. Gorno-Tempini ML, Dronkers NF, Rankin KP, et al. Cognition and anatomy in three variants of primary progressive aphasia. Ann Neurol. 2004; 55(3):335-346. [PubMed: 14991811]

11*. Mesulam MM, Wieneke C, Thompson C, Rogalski E, Weintraub S. Quantitative classification of primary progressive aphasia at early and mild impairment stages. Brain. 2012; 135(Pt 5):15371553. State-of-the art in quantifying symptoms of PPA and differential diagnosis of variants. [PubMed: 22525158]

12. Rogalski E, Cobia D, Harrison TM, Wieneke C, Weintraub S, Mesulam MM. Progression of language decline and cortical atrophy in subtypes of primary progressive aphasia. Neurology. 2011; 76(21):1804-1810. [PubMed: 21606451]

13. Hillis AE, Tuffiash E, Caramazza A. Modality-specific deterioration in naming verbs in nonfluent primary progressive aphasia. J Cogn Neurosci. 2002; 14(7):1099-1108. [PubMed: 12419132]

$14 *$. Hillis AE, Oh S, Ken L. Deterioration of naming nouns versus verbs in primary progressive aphasia. Ann Neurol. 2004; 55(2):268-275. Deficits in noun and verb production in the three variants of PPA. [PubMed: 14755731]

15. Hillis AE, Heidler-Gary J, Newhart M, Chang S, Ken L, Bak TH. Naming and comprehension in primary progressive aphasia: The influence of grammatical word class. Aphasiology. 2006; 20(0204):246-256.

16. Gorno-Tempini ML, Ogar JM, Brambati SM, et al. Anatomical correlates of early mutism in progressive nonfluent aphasia. Neurology. 2006; 67(10):1849-1851. [PubMed: 16931509]

17. Gorno-Tempini ML, Murray RC, Rankin KP, Weiner MW, Miller BL. Clinical, cognitive and anatomical evolution from nonfluent progressive aphasia to corticobasal syndrome: A case report. Neurocase. 2004; 10(6):426-436. [PubMed: 15788282]

18. Josephs KA, Whitwell JL, Duffy JR, et al. Progressive aphasia secondary to alzheimer disease vs FTLD pathology. Neurology. 2008; 70(1):25-34. [PubMed: 18166704]

19. Wilson SM, Galantucci S, Tartaglia MC, et al. Syntactic processing depends on dorsal language tracts. Neuron. 2011; 72(2):397-403. [PubMed: 22017996] 
20. Hurley RS, Paller KA, Rogalski EJ, Mesulam MM. Neural mechanisms of object naming and word comprehension in primary progressive aphasia. J Neurosci. 2012; 32(14):4848-4855. [PubMed: 22492040]

21. Seeley WW, Bauer AM, Miller BL, et al. The natural history of temporal variant frontotemporal dementia. Neurology. 2005; 64(8):1384-1390. [PubMed: 15851728]

22. Seeley WW, Matthews BR, Crawford RK, et al. Unravelling bolero: Progressive aphasia, transmodal creativity and the right posterior neocortex. Brain. 2008; 131(Pt 1):39-49. [PubMed: 18057074]

23*. Gorno-Tempini ML, Brambati SM, Ginex V, et al. The logopenic/phonological variant of primary progressive aphasia. Neurology. 2008; 71(16):1227-1234. Detailed description and characteristics of the logopenic variant of PPA. [PubMed: 18633132]

24. Rohrer JD, Caso F, Mahoney C, et al. Patterns of longitudinal brain atrophy in the logopenic variant of primary progressive aphasia. Brain Lang. 2013; 127(2):121-126. [PubMed: 23395096]

25. Beeson PM, King RM, Bonakdarpour B, Henry ML, Cho H, Rapcsak SZ. Positive effects of language treatment for the logopenic variant of primary progressive aphasia. J Mol Neurosci. 2011; 45(3):724-736. [PubMed: 21710364]

26. Henry ML, Beeson PM, Rapcsak SZ. Treatment for anomia in semantic dementia. Semin Speech Lang. 2008; 29(1):60-70. [PubMed: 18348092]

27. Henry ML, Beeson PM, Alexander GE, Rapcsak SZ. Written language impairments in primary progressive aphasia: A reflection of damage to central semantic and phonological processes. $\mathbf{J}$ Cogn Neurosci. 2011

$28 * *$. Rapp B, Glucroft B. The benefits and protective effects of behavioural treatment for dysgraphia in a case of primary progressive aphasia. Aphasiology. 2009; 23(2):236-265. Behavioral treatment of written language lexical deficits in a case of logopenic PPA. [PubMed: 21603153]

$29 * *$. Tsapkini K, Hillis AE. Spelling intervention in post-stroke aphasia and primary progressive aphasia. Behav Neurol. 2013; 26(1-2):55-66. Commonalities and differences in the treatment trajectory of spelling deficits between a case of post-stroke aphasia and a case of PPA. [PubMed: 22713403]

30. Budd MA, Kortte K, Cloutman L, et al. The nature of naming errors in primary progressive aphasia versus acute post-stroke aphasia. Neuropsychology. 2010; 24(5):581-589. [PubMed: 20804246]

31*. Sepelyak K, Crinion J, Molitoris J, et al. Patterns of breakdown in spelling in primary progressive aphasia. Cortex. 2011; 47(3):342-352. Spelling deficits in the three variants of PPA and neural correlates. [PubMed: 20060967]

32. Ellis, AW. Reading, Writing and Dyslexia: A Cognitive Analysis. Psychology Press; 1993.

33. Ellis, AW.; Young, AW. Human Cognitive Neuropsychology. Hillsdale, NJ: Lawrence Erlbaum Associates; 1988.

34. Kiran S, Thompson CK, Hashimoto N. Training grapheme to phoneme conversion in patients with oral reading and naming deficits: A model-based approach. Aphasiology. 2001; 15(9):855-876.

35. Kiran S. Training phoneme to grapheme conversion for patients with written and oral production deficits: A model-based approach. Aphasiology. 2005; 19(1):53-76.

36. Beeson PM, Rising K, Volk J. Writing treatment for severe aphasia: Who benefits? J Speech Lang Hear Res. 2003; 46(5):1038-1060. [PubMed: 14575342]

37. Beeson PM, Egnor H. Combining treatment for written and spoken naming. J Int Neuropsychol Soc. 2006; 12(6):816-827. [PubMed: 17064445]

38. Best W, Nickels L. From theory to therapy in aphasia: Where are we now and where to next? Neuropsychological Rehabilitation. 2000; 10(3):231-247.

39. Bastiaanse R, Bosje M, Franssen M. Deficit-oriented treatment of word-finding problems: Another replication. Aphasiology. 1996; 10(4):363-383.

40. Hillis AE. Efficacy and generalization of treatment for aphasic naming errors. Arch Phys Med Rehabil. 1989; 70(8):632-636. [PubMed: 2764694]

41. DeDe G, Parris D, Waters G. Teaching self-cues: A treatment approach for verbal naming. Aphasiology. 2003; 17(5):465-480. 
42*. Grossman M. Progressive aphasic syndromes: Clinical and theoretical advances. Curr Opin Neurol. 2002; 15(4):409-413. Classification of aphasic symptoms in PPA. [PubMed: 12151836]

43. Thompson CK, Lukic S, King MC, Mesulam MM, Weintraub S. Verb and noun deficits in strokeinduced and primary progressive aphasia: The northwestern naming battery(). Aphasiology. 2012; 26(5):632-655. [PubMed: 23188949]

44. Corbett F, Jefferies E, Ehsan S, Lambon Ralph MA. Different impairments of semantic cognition in semantic dementia and semantic aphasia: Evidence from the non-verbal domain. Brain. 2009; 132(Pt 9):2593-2608. [PubMed: 19506072]

45**. Croot K, Nickels L, Laurence F, Manning M. Impairment- and activity/participation-directed interventions in progressive language impairment: Clinical and theoretical issues. Aphasiology. 2009; 23(2):125-160. Examples and directions for treatment of aphasic symptoms in PPA.

46**. Graham KS, Patterson K, Pratt KH, Hodges JR. Relearning and subsequent forgetting of semantic category exemplars in a case of semantic dementia. Neuropsychology. 1999; 13(3): 359-380. Behavioral treatment of semantic deficits in a case of semantic variant PPA. [PubMed: 10447298]

47**. Henry ML, Beeson PM, Rapcsak SZ. Treatment for lexical retrieval in progressive aphasia. Aphasiology. 2008; 22(7-8):826-838. Behavioral treatment of oral and written production in PPA. [PubMed: 20862210]

48**. Jokel R, Rochon E, Leonard C. Treating anomia in semantic dementia: Improvement, maintenance, or both? Neuropsychol Rehabil. 2006; 16(3):241-256. Anomia treatment in semantic variant PPA. [PubMed: 16835150]

49**. Jokel R, Rochon E, Anderson ND. Errorless learning of computer-generated words in a patient with semantic dementia. Neuropsychol Rehabil. 2010; 20(1):16-41. Errorless learning as a treatment approach in semantic variant PPA. [PubMed: 19504403]

50. Jokel R, Anderson ND. Quest for the best: Effects of errorless and active encoding on word relearning in semantic dementia. Neuropsychol Rehabil. 2012; 22(2):187-214. [PubMed: 22250922]

$51^{* *}$. Marcotte K, Ansaldo AI. The neural correlates of semantic feature analysis in chronic aphasia: Discordant patterns according to the etiology. Semin Speech Lang. 2010; 31(1):52-63. Neural network analysis of treatment effects in chronic post-stroke aphasia: relationship between language and control networks before and after intervention. [PubMed: 20221954]

$52 * *$. McNeil M, Small S, Masterson RJ, Fossett T. Behavioural and pharmacological treatment of lexical-semantic deficits in a single patient with primary progressive aphasia. American Journal of Speech-Language Pathology. 1995; 4(4):76-87. Effects of behavioral treatment of cueing hierarchy of predicative adjectives combined with dextroamphetamine treatment.

53. Schneider S, Thompson C, Luring B. Effects of verbal plus gestural matrix training on sentence production in a patient with primary progressive aphasia. aphasiology. Aphasiology. 1996; 10(3): 297-317.

54. Henry ML, Beeson PM, Alexander GE, Rapcsak SZ. Written language impairments in primary progressive aphasia: A reflection of damage to central semantic and phonological processes. $\mathbf{J}$ Cogn Neurosci. 2012; 24(2):261-275. [PubMed: 22004048]

55**. Newhart M, Davis C, Kannan V, Heidler-Gary J, Cloutman L, Hillis AE. Therapy for naming deficits in two variants of primary progressive aphasia. Aphasiology. 2009; 23(7-8):823-834. Variation of therapeutic approaches and results in a case of semantic and a case of logopenic PPA.

56**. Beeson PM, King RM, Bonakdarpour B, Henry ML, Cho H, Rapcsak SZ. Positive effects of language treatment for the logopenic variant of primary progressive aphasia. J Mol Neurosci. 2011; 45(3):724-736. Improvement of oral and written naming after treatment in logopenic variant PPA. [PubMed: 21710364]

57**. Henry ML, Beeson PM, Rapcsak SZ. Treatment for anomia in semantic dementia. Semin Speech Lang. 2008; 29(1):60-70. Improvement in naming in semantic variant PPA. [PubMed: 18348092]

58. Meyer A, Getz H, Snider S, et al. Remediation and prophylaxis of anomia in primary progressive aphasia. Procedia Soc Behav Sci. 2013; 94:275-276. [PubMed: 25101147] 
59**. Baker JM, Rorden C, Fridriksson J. Using transcranial direct-current stimulation to treat stroke patients with aphasia. Stroke. 2010; 41(6):1229-1236. Seminal study on using tDCS in poststroke remediation of naming deficits. [PubMed: 20395612]

60**. Fridriksson J, Richardson JD, Baker JM, Rorden C. Transcranial direct current stimulation improves naming reaction time in fluent aphasia: A double-blind, sham-controlled study. Stroke. 2011; 42(3):819-821. Follow-up study on RT improvement in naming in post-stroke aphasia using tDCS. [PubMed: 21233468]

61**. Dmochowski JP, Datta A, Huang Y, et al. Targeted transcranial direct current stimulation for rehabilitation after stroke. Neuroimage. 2013; 75:12-19. Optimization of electrode placement for tDCS in naming remediation in post-stroke aphasia. [PubMed: 23473936]

62**. Boggio PS, Khoury LP, Martins DC, Martins OE, de Macedo EC, Fregni F. Temporal cortex direct current stimulation enhances performance on a visual recognition memory task in alzheimer disease. J Neurol Neurosurg Psychiatry. 2009; 80(4):444-447. tDCS contributed to longer lasting visual memories in AD than sham. [PubMed: 18977813]

$63^{* *}$. Boggio PS, Valasek CA, Campanha C, et al. Non-invasive brain stimulation to assess and modulate neuroplasticity in alzheimer's disease. Neuropsychol Rehabil. 2011; 21(5):703-716. tDCS modulates neuroplasticity in AD. [PubMed: 21942868]

64. Hansen N. Action mechanisms of transcranial direct current stimulation in alzheimer's disease and memory loss. Front Psychiatry. 2012; 3:48. [PubMed: 22615703]

$65^{* *}$. Stagg CJ, Nitsche MA. Physiological basis of transcranial direct current stimulation. Neuroscientist. 2011; 17(1):37-53. tDCS acts at the synaptic level of neuronal transmission. [PubMed: 21343407]

66. Nardone R, Bergmann J, Christova M, et al. Effect of transcranial brain stimulation for the treatment of alzheimer disease: A review. Int J Alzheimers Dis. 2012; 2012:687909. [PubMed: 22114748]

67. Monte-Silva K, Kuo MF, Hessenthaler S, et al. Induction of late LTP-like plasticity in the human motor cortex by repeated non-invasive brain stimulation. Brain Stimul. 2013; 6(3):424-432. [PubMed: 22695026]

68. Price CJ, Mechelli A. Reading and reading disturbance. Curr Opin Neurobiol. 2005; 15(2):231238. [PubMed: 15831408]

69. Meinzer M, Lindenberg R, Darkow R, Ulm L, Copland D, Floel A. Transcranial direct current stimulation and simultaneous functional magnetic resonance imaging. J Vis Exp. 2014; (86) $10.3791 / 51730$

70**. Baker JM, Rorden C, Fridriksson J. Using transcranial direct-current stimulation to treat stroke patients with aphasia. Stroke. 2010; 41(6):1229-1236. Specifications of using tDCS in poststroke aphasia. [PubMed: 20395612]

71. Reis J, Fritsch B. Modulation of motor performance and motor learning by transcranial direct current stimulation. Curr Opin Neurol. 2011; 24(6):590-596. [PubMed: 21968548]

$72 * *$. Monte-Silva K, Kuo MF, Hessenthaler S, et al. Induction of late LTP-like plasticity in the human motor cortex by repeated non-invasive brain stimulation. Brain Stimul. 2013; 6(3):424432. The mechanism of action of repeated tDCS is by inducing LTP-like plasticity. [PubMed: 22695026]

73. Reis J, Schambra HM, Cohen LG, et al. Noninvasive cortical stimulation enhances motor skill acquisition over multiple days through an effect on consolidation. Proc Natl Acad Sci U S A. 2009; 106(5):1590-1595. [PubMed: 19164589]

74. Meinzer M, Jahnigen S, Copland DA, et al. Transcranial direct current stimulation over multiple days improves learning and maintenance of a novel vocabulary. Cortex. 2014; 50:137-147. [PubMed: 23988131]

75**. Fridriksson J, Richardson JD, Baker JM, Rorden C. Transcranial direct current stimulation improves naming reaction time in fluent aphasia: A double-blind, sham-controlled study. Stroke. 2011; 42(3):819-821. Treatment of patients with posterior lesions using tDCS. [PubMed: 21233468]

76. Fridriksson J, Hubbard HI, Hudspeth SG. Transcranial brain stimulation to treat aphasia: A clinical perspective. Semin Speech Lang. 2012; 33(3):188-202. [PubMed: 22851341] 
77**. Boggio PS, Valasek CA, Campanha C, et al. Non-invasive brain stimulation to assess and modulate neuroplasticity in alzheimer's disease. Neuropsychol Rehabil. 2011; 21(5):703-716. Establishing tDCS as a method to modulate neuronal plasticity in neural degeneration. [PubMed: 21942868]

78**. Boggio PS, Ferrucci R, Mameli F, et al. Prolonged visual memory enhancement after direct current stimulation in alzheimer's disease. Brain Stimul. 2012; 5(3):223-230. tDCS improves visual memories in patients with AD. [PubMed: 21840288]

79**. Boggio PS, Ferrucci R, Rigonatti SP, et al. Effects of transcranial direct current stimulation on working memory in patients with parkinson's disease. J Neurol Sci. 2006; 249(1):31-38. tDCS improves working memory in patients with Parkinson's disease. [PubMed: 16843494]

80**. Boggio PS, Ferrucci R, Mameli F, et al. Prolonged visual memory enhancement after direct current stimulation in alzheimer's disease. Brain Stimul. 2012; 5(3):223-230. tDCS improves visual memory in AD. [PubMed: 21840288]

81. Polania R, Paulus W, Nitsche MA. Reorganizing the intrinsic functional architecture of the human primary motor cortex during rest with non-invasive cortical stimulation. PLoS One. 2012; 7(1):e30971. [PubMed: 22303478]

82. Polania R, Paulus W, Antal A, Nitsche MA. Introducing graph theory to track for neuroplastic alterations in the resting human brain: A transcranial direct current stimulation study. Neuroimage. 2011; 54(3):2287-2296. [PubMed: 20932916]

83. Polania R, Paulus W, Nitsche MA. Modulating cortico-striatal and thalamo-cortical functional connectivity with transcranial direct current stimulation. Hum Brain Mapp. 2011

84. Fritsch B, Reis J, Martinowich K, et al. Direct current stimulation promotes BDNF-dependent synaptic plasticity: Potential implications for motor learning. Neuron. 2010; 66(2):198-204. [PubMed: 20434997]

85. Stagg CJ, Nitsche MA. Physiological basis of transcranial direct current stimulation. Neuroscientist. 2011; 17(1):37-53. [PubMed: 21343407]

86. Fiori V, Coccia M, Marinelli CV, et al. Transcranial direct current stimulation improves word retrieval in healthy and nonfluent aphasic subjects. J Cogn Neurosci. 2011; 23(9):2309-2323. [PubMed: 20946060]

87**. Marangolo P, Marinelli CV, Bonifazi S, et al. Electrical stimulation over the left inferior frontal gyrus (IFG) determines long-term effects in the recovery of speech apraxia in three chronic aphasics. Behav Brain Res. 2011; 225(2):498-504. tDCS improves outcome of apraxia of speech treatment more than sham in post-stroke aphasia. [PubMed: 21856336]

88. Fiori V, Cipollari S, Di Paola M, Razzano C, Caltagirone C, Marangolo P. tDCS stimulation segregates words in the brain: Evidence from aphasia. Front Hum Neurosci. 2013; 7:269. [PubMed: 23785323]

89**. Marangolo P, Fiori V, Calpagnano MA, et al. tDCS over the left inferior frontal cortex improves speech production in aphasia. Front Hum Neurosci. 2013; 7:539. tDCS improves speech production more than sham in post-stroke aphasia. [PubMed: 24046740]

90. Chrysikou EG, Hamilton RH. Noninvasive brain stimulation in the treatment of aphasia: Exploring interhemispheric relationships and their implications for neurorehabilitation. Restor Neurol Neurosci. 2011

91**. Monti A, Cogiamanian F, Marceglia S, et al. Improved naming after transcranial direct current stimulation in aphasia. J Neurol Neurosurg Psychiatry. 2008; 79(4):451-453. Cathodal tDCS improves naming in post-stroke aphasia. [PubMed: 18096677]

92**. Datta A, Baker JM, Bikson M, Fridriksson J. Individualized model predicts brain current flow during transcranial direct-current stimulation treatment in responsive stroke patient. Brain Stimul. 2011; 4(3):169-174. tDCS Electrode placement according to individualized computational models of current flow. [PubMed: 21777878]

93**. Jo JM, Kim YH, Ko MH, Ohn SH, Joen B, Lee KH. Enhancing the working memory of stroke patients using tDCS. Am J Phys Med Rehabil. 2009; 88(5):404-409. Working memory is improved with tDCS post-stroke. [PubMed: 19620953]

Curr Treat Options Neurol. Author manuscript; available in PMC 2015 October 10. 
94. Marangolo P, Marinelli CV, Bonifazi S, et al. Electrical stimulation over the left inferior frontal gyrus (IFG) determines long-term effects in the recovery of speech apraxia in three chronic aphasics. Behav Brain Res. 2011; 225(2):498-504. [PubMed: 21856336]

95. Fiori V, Coccia M, Marinelli CV, et al. Transcranial direct current stimulation improves word retrieval in healthy and nonfluent aphasic subjects. J Cogn Neurosci. 2011; 23(9):2309-2323. [PubMed: 20946060]

96. Kuo MF, Paulus W, Nitsche MA. Therapeutic effects of non-invasive brain stimulation with direct currents (tDCS) in neuropsychiatric diseases. Neuroimage. 2014; 85(Pt 3):948-960. [PubMed: 23747962]

97. Brunoni AR, Nitsche MA, Bolognini N, et al. Clinical research with transcranial direct current stimulation (tDCS): Challenges and future directions. Brain Stimul. 2011

98**. Ferrucci R, Mameli F, Guidi I, et al. Transcranial direct current stimulation improves recognition memory in alzheimer disease. Neurology. 2008; 71(7):493-498. tDCS improves recognition in AD. [PubMed: 18525028]

99**. Huey ED, Probasco JC, Moll J, et al. No effect of DC brain polarization on verbal fluency in patients with advanced frontotemporal dementia. Clin Neurophysiol. 2007; 118(6):1417-1418. No effects of tDCS after one application. [PubMed: 17452012]

$100^{* *}$. Cotelli M, Manenti R, Petesi M, et al. Treatment of primary progressive aphasias by transcranial direct current stimulation combined with language training. J Alzheimers Dis. 2014; 39(4):799-808. tDCS improves naming in PPA. [PubMed: 24296814]

101**. Tsapkini K, Frangakis C, Gomez Y, Davis C, Hillis AE. Augmentation of spelling therapy with transcranial direct current stimulation in primary progressive aphasia: Preliminary results and challenges. Aphasiology. 2014; 28(8-9):1112-1130. tDCS improves spelling in PPA: effects are generalized and last longer than sham. [PubMed: 26097278]

102. Antal A, Terney D, Poreisz C, Paulus W. Towards unravelling task-related modulations of neuroplastic changes induced in the human motor cortex. Eur J Neurosci. 2007; 26(9):26872691. [PubMed: 17970738]

103. Andrews SC, Hoy KE, Enticott PG, Daskalakis ZJ, Fitzgerald PB. Improving working memory: The effect of combining cognitive activity and anodal transcranial direct current stimulation to the left dorsolateral prefrontal cortex. Brain Stimul. 2011; 4(2):84-89. [PubMed: 21511208]

104. Segrave RA, Arnold S, Hoy K, Fitzgerald PB. Concurrent cognitive control training augments the antidepressant efficacy of tDCS: A pilot study. Brain Stimul. 2014; 7(2):325-331. [PubMed: 24486425]

105. Antal A, Polania R, Schmidt-Samoa C, Dechent P, Paulus W. Transcranial direct current stimulation over the primary motor cortex during fMRI. Neuroimage. 2011; 55(2):590-596. [PubMed: 21211569]

106. Antal A, Paulus W, Nitsche MA. Electrical stimulation and visual network plasticity. Restor Neurol Neurosci. 2011; 29(6):365-374. [PubMed: 22124032]

107. Finocchiaro C, Maimone M, Brighina F, Piccoli T, Giglia G, Fierro B. A case study of primary progressive aphasia: Improvement on verbs after rTMS treatment. Neurocase. 2006; 12(6):317321. [PubMed: 17182394]

108**. Trebbastoni A, Raccah R, de Lena C, Zangen A, Inghilleri M. Repetitive deep transcranial magnetic stimulation improves verbal fluency and written language in a patient with primary progressive aphasia-logopenic variant (LPPA). Brain Stimul. 2013; 6(4):545-553. TMS improves verbal fluency and written language in PPA. [PubMed: 23122915]

109**. Cotelli M, Manenti R, Alberici A, et al. Prefrontal cortex rTMS enhances action naming in progressive non-fluent aphasia. Eur J Neurol. 2012; 19(11):1404-1412. rTMS improves action naming in non-fluent variant PPA. [PubMed: 22435956]

110. Decker DA, Heilman KM. Steroid treatment of primary progressive aphasia. Arch Neurol. 2008; 65(11):1533-1535. [PubMed: 19001174]

111. Shankle WR, Hara J, Bjornsen L, et al. Omental therapy for primary progressive aphasia with tau negative histopathology: 3 year study. Neurol Res. 2009; 31(7):766-769. [PubMed: 19138468]

Curr Treat Options Neurol. Author manuscript; available in PMC 2015 October 10. 
112**. Cotelli M, Manenti R, Brambilla M, et al. Anodal tDCS during face-name associations memory training in alzheimer's patients. Front Aging Neurosci. 2014; 6:38. Anodal tDCS improves memory associations in AD. [PubMed: 24678298] 


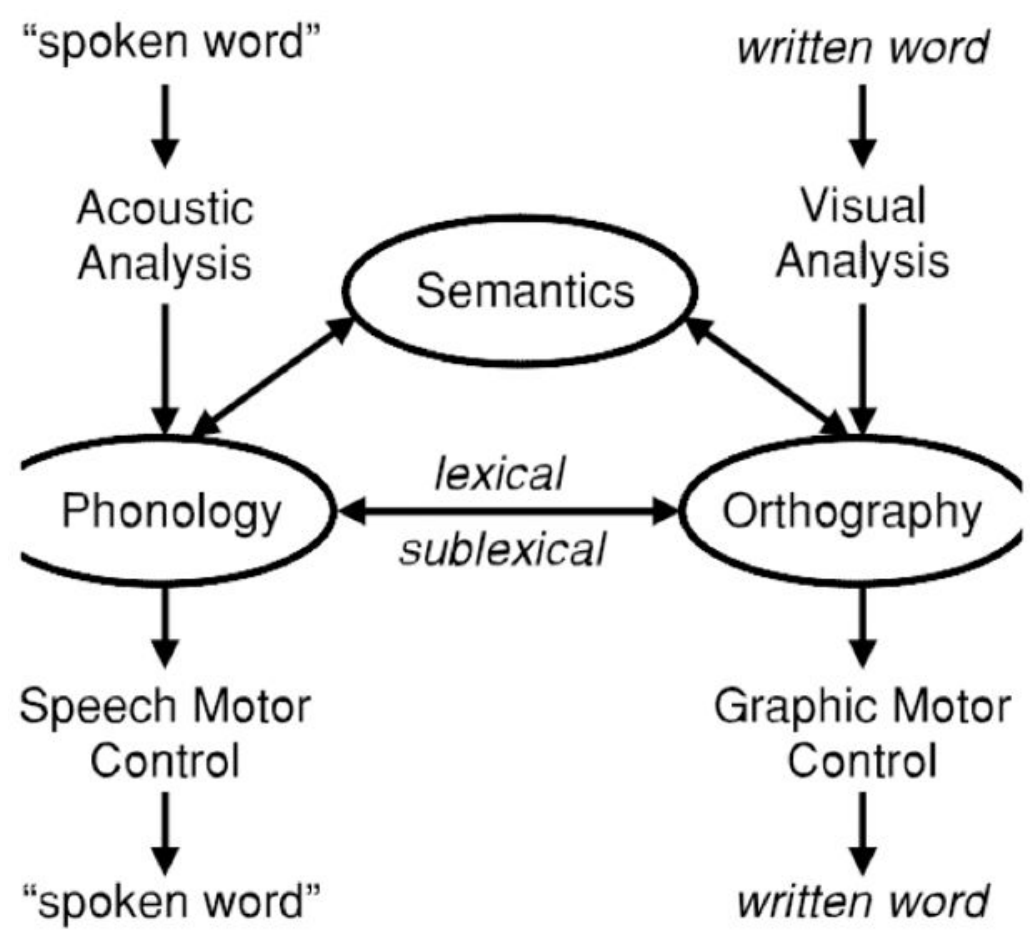

Figure 1.

Interactive model of lexical processing 